\title{
Biopsia prostática ecodirigida: comparación de dos métodos anestésicos
}

\author{
C. Conde Redondo, D. Alonso Fernández, A. Robles Samaniego, N. Del Valle González, \\ F. Castroviejo Royo, C. Delgado Marcos, A. Rodríguez Toves, J.M. Martínez-Sagarra Oceja
}

Servicio de Urología. Hospital Río Hortega. Valladolid.

Actas Urol Esp 2006; 30 (2): 134-138

\section{RESUMEN}

BIOPSIA PROSTÁTICA ECODIRIGIDA: COMPARACIÓN DE DOS MÉTODOS ANESTÉSICOS

Introducción: La intención de este estudio es comparar la efectividad de dos técnicas analgésicas, una oral frente al bloqueo con anestesia local de los nervios periprostáticos, en la realización de la biopsia prostática ecodirigida.

Material y métodos: Un Total de 200 pacientes se han randomizado en dos grupos. Grupo I: 100 pacientes tratados con metamizol y morfina oral 30 minutos antes de la realización de la biopsia. Grupo II: 100 pacientes a los que se los ha anestesiado con mepivacaína al $2 \%$ en las bandeletas. Previamente a ambos grupos de pacientes se les suministró bromacepán $3 \mathrm{mg} 30$ minutos antes de la prueba. Después de la biopsia a los pacientes se les pidió que graduaran su dolor mediante una escala analógica visual, graduada de 0 (no dolor) a 10 dolor insoportable. Los datos obtenidos se manejaron estadísticamente con el test T de Student.

Resultados: Ambos grupos no presentaron diferencias significativas con respecto a la edad, PSA y volumen prostático. Se obtuvieron en el grupo I, 3 biopsias por lóbulo $(3 \pm 1,3)$, y en el II grupo $5(5 \pm 1,2)$. En éste grupo el $95 \%$ de los pacientes no experimentaron dolor alguno tras la biopsia (VAS = 0), el 2\% un dolor moderado (VAS=5-6), y el 3\% un dolor intenso (VAS=7-8). Los pacientes del grupo I refirieron ningún dolor en 12,5\%, dolor moderado el 42, 4\%, y dolor intenso el $20 \%$. Encontrándose diferencia significativa entre ambos grupos $(\mathrm{p} \leq 0,05)$ con respecto al dolor, pero no con respecto a las complicaciones referidas.

Conclusión: La biopsia de próstata ecodirigida es una prueba muy bien tolerada bajo anestesia local con mepivacaína, disminuyendo el dolor asociado a la biopsia, lo cual permite realizar en el mismo acto una mayor toma de cilindros.

Palabras clave: Biopsia prostática. Cáncer de próstata. Anestesia.

\section{ABSTRACT \\ TRUS-GUIDED BIOPSY: COMPARISON OF TWO ANESTHETIC METHODS}

Purpose: The aim of the present study is to compare two analgesic techniques for ultrasound transrectal biopsy. Oral analgesia vs periprosthetic nerve blockade with $2 \%$ mevicaine.

Patients and methods: A total of 200 patients were randomized prospectively into 2 groups, namely group I: 100 patients treated with metamizol, oral morphine 30 minutes before the procedure, and group II: 100 patients anesthesied with periprosthetic nerve blockade with $2 \%$ mepivacaine. Both groups were treated with bromacepán 3 mg 30 minutes before the biopsy. The first intention was to obtain 10 core TRUS-guided biopsy in all patients underwent. After the procedure, a ten visual analogue pain score (VAS) from $0=$ no discomfort to $10=$ severe pain was administered to the biopsied patients and a global estimation of pain associated with the procedure was obtained. Test $\mathrm{T}$ de Student was used for statistical analysis.

Results: There were no significant differences in age, PSA and prostate volume. 3 core TRUS-guided biopsy were obtain in group I $(3 \pm 1,3)$, and 10 in group II $(5 \pm 1,2)$ In the periprosthetic block group (II) $95 \%$ of patients referred no pain after the procedure (VAS $=0$ ), $2 \%$ middle pain (VAS=5-6) and $3 \%$ strong pain (VAS=7-8); while patients in group I referred $12,5 \%$ no pain , $42,4 \%$ middle pain, $20 \%$ strong pain. The level of pain reported by this group of patients was significantly different from those reported by patients who performed prostate biopsy with periprosthetic nerve blockade. ( $\mathrm{p} \leq 0.05)$. There were no significant differences in major complications.

Conclusions: The use of bilateral periprosthetic block with mepivacaine is a very effective and useful technique, well tolerated by the patient, which almost completely abolishes the pain and discomfort associated with the prostate biopsy procedure. And also allows increase the number of cores.

Key words: Prostate biopsy. Prostate cancer. Anaesthesia. 
$\mathrm{E}$ cáncer de próstata representa uno de los problemas de salud más importantes del mundo occidental. La detección del mismo se lleva a cabo por medio de la biopsia de próstata, que es una prueba dolorosa y cruenta. Alrededor del 60-90\% ${ }^{1}$ de los pacientes refieren molestias al realizarles la biopsia de próstata, que pueden ser leves, dolor severo, e incluso producir reacciones vasovagales. El dolor del paciente implica una clara reducción del número de biopsias obtenidas durante la prueba, y por tanto una disminución de la tasa de detección del cáncer de próstata.

En enero del 2001, sólo el 11\% de los urólogos que realizaban esta técnica, se preocupaban por disminuir el dolor producido por la misma mediante el bloqueo de los nervios periprostáticos con anestésico local ${ }^{2}$. Afortunadamente durante los últimos años se ha despertado un creciente interés por disminuir el disconfort de los pacientes.

En este artículo comparamos la efectividad de dos técnicas analgésicas, una oral frente a otra local en la realización de la biopsia prostática.

\section{MATERIAL Y MÉTODOS}

Durante el año 2004, los pacientes sometidos a biopsia prostática eco-dirigida con ecógrafo Hitachi EV525 y transductor end-fire 7,0 mHz, fueron divididos en dos grupos de una manera randomizada y prospectiva; 100 pacientes fueron sometidos a la biopsia bajo analgesia oral (grupo I), consistente en Bromacepan $3 \mathrm{mg}$ (Lexatin ${ }^{\circledR}$ ), Metamizol $\left(\right.$ Nolotil $^{\circledR}$ ), morfina oral (Sevredol ${ }^{\circledR}$ ), 30 minutos antes de la intervención. A otros 100 pacientes, (grupo II), se le realizó la biopsia bajo anestesia local con mepivacaína al 2\% $10 \mathrm{ml}$ inyectado en el ángulo que en el corte longitudinal forman las bandeletas con la próstata, tal como describe Soloway ${ }^{3}$. Esta es la zona de proyección de las bandeletas. Inicialmente se forma ahí el habón y posteriormente se extrae la aguja de punción de $22 \mathrm{G}$ hasta formar otro pequeño habón en la zona del ápex prostático (Figs. 1, 2 y 3), dejando actuar el anestésico durante 10 minutos. Previamente estos pacientes también habían sido tratados con Bromacepan $3 \mathrm{mg}$ media hora antes de la intervención.

Posteriormente a la biopsia se invitó a los pacientes a valorar el grado de dolor que habían

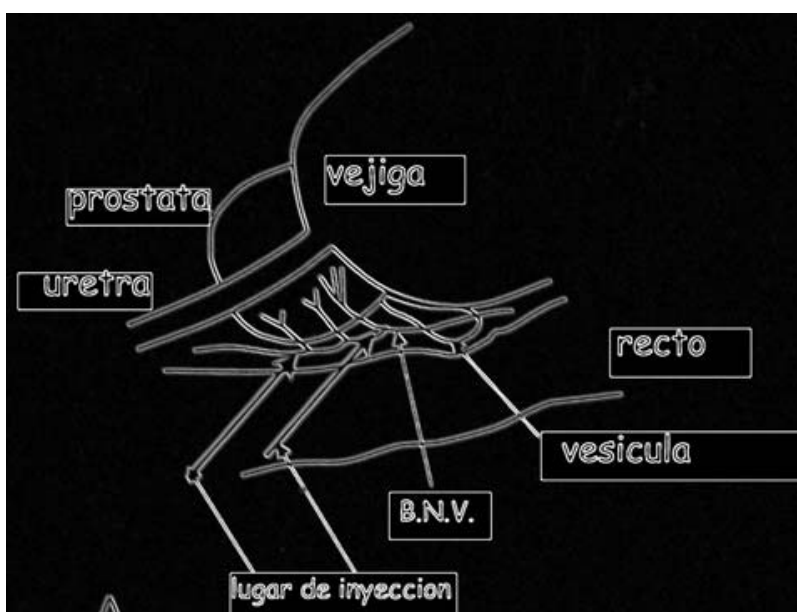

FIGURA 1. Esquema de las bandeletas neurovasculares.

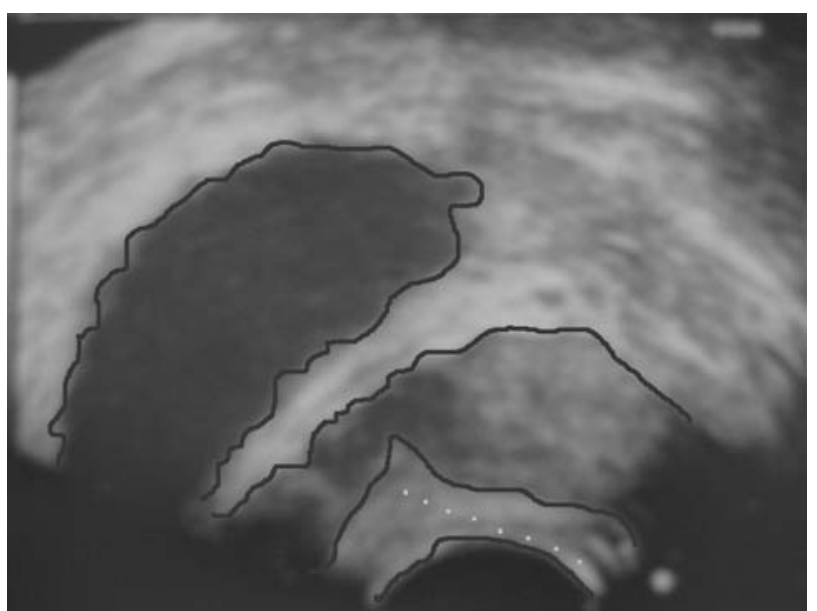

FIGURA 2. Ángulo formado entre la vesícula seminal y la próstata.

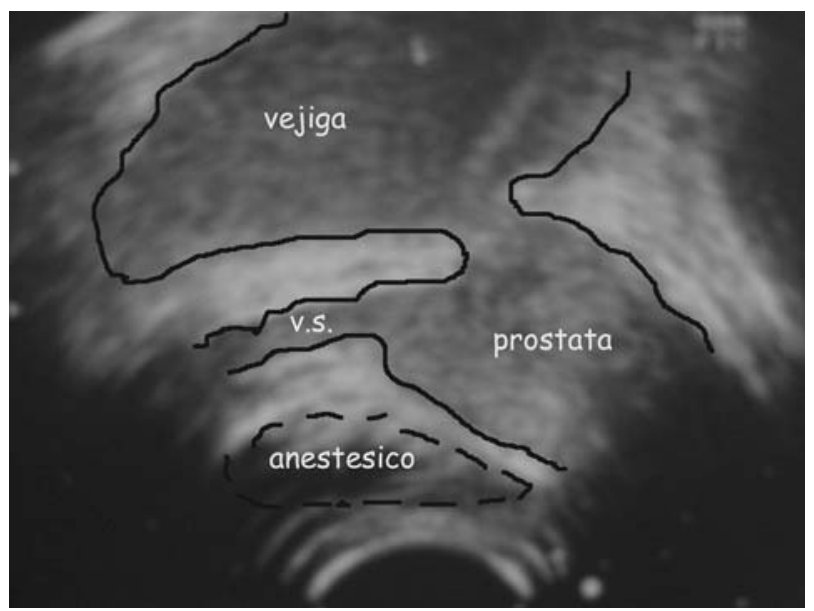

FIGURA 3. Habón formado de anestésico local entre el recto y la próstata. 
sufrido, mediante una escala analógica visual de dolor, que gradúa el dolor de 1 al 10. Así hemos clasificado el dolor en leve (grado 1,2,3), moderado $(4,5,6)$ e intenso, si puntuaron en la escala analógica visual por encima de 6 .

Posteriormente les dimos un cuestionario autoadministrable en el que reflejaban el tipo de complicaciones que sufrieron tras la biopsia: hematuria, fiebre, rectorragia, RAO etc.

Los datos obtenidos se manejaron estadísticamente con el test $\mathrm{T}$ de Student, (test de comparación de medias) siendo estadísticamente significativa la diferencia entre grupos para una $p \leq 0,05$.

\section{RESULTADOS}

Ambos grupos I y II fueron homogéneos, con respecto a la edad, volumen prostático y valor de PSA.

El número de cilindros prostáticos obtenidos durante la biopsia fue mayor en el grupo II, 5 cilindros de cada lóbulo prostático de media $(5 \pm 1,2)$, frente a al grupo I con una media de 3 cilindros por lóbulo $(3 \pm 1,3)$. Con respecto al número de complicaciones obtenidas tras la biopsia, no se encontró diferencia significativa entre ambos grupos para las complicaciones tardías: fiebre $3,3 \%$, hematuria $68,4 \%$, rectorragia $28,6 \%$, hemospermia $41,1 \%$, disuria $26,6 \%$ y RAO $1 \%$. Acudieron a urgencias el 3,9\%, y necesitaron ingreso el 3\% de los mismos. Sin embargo, sí que encontramos diferencia significativa entre ambos grupos respecto a la complicación precoz más frecuente; la reacción vasovagal (mareos, hipotensión, vómitos). El $20 \%$ de los pacientes que fueron biopsiados con analgesia oral presentaron una reacción vagal, si bien es cierto de pequeña intensidad y en ningún caso precisaron ingreso, frente al grupo de la anestesia local, en el que sólo se produjo una reacción vasovagal (1\%).

Con respecto al dolor reflejado por los pacientes del grupo I, destaca que no sufrieron dolor el $12,5 \%$, un dolor leve el $42,4 \%$, e intenso el $20 \%$, no siendo finalmente capaces de graduar el dolor el $24,7 \%$ de los encuestados. En el grupo II presentaron dolor intenso el $2 \%$ de los pacientes, dolor leve el 3\% y llama la atención que el 95\% de ellos no refiere presentar dolor alguno. Por lo que encontramos una clara diferencia significativa entre ambos grupos $(\leq 0,05)$.

\section{DISCUSIÓN}

La indicación de la biopsia de próstata tras el estudio de múltiples test de despistaje se puede resumir en:

- pacientes con tacto rectal sospechoso.

- varones menores de 50 años con PSA $\geq 2,5$ $\mathrm{ng} / \mathrm{ml}$ y/o índice PSA libre/total $\leq 18 \%$.

- varones entre 50 y 60 años con PSA $\geq 3,5$ $\mathrm{ng} / \mathrm{ml}$ y/o índice PSA libre/total $\leq 18 \%$.

- varones mayores de 60 años con PSA $\geq 4$ $\mathrm{ng} / \mathrm{ml}$ y/o índice PSA libre/total $\leq 15 \%$.

Y la indicación de repetición de biopsia se resume en

- PSA $\geq 10 \mathrm{ng} / \mathrm{ml}$,

- PIN de alto grado,

- Velocidad de PSA $\geq 1 \mathrm{ng} / \mathrm{ml} /$ año,

- índice PSA libre/total $\leq 15-20 \%{ }^{4}$.

Parece ser que la tradicional biopsia por sextantes está en el momento actual obsoleta, y ahora la tendencia es a aumentar el número de biopsias y a realizarlas en la zona más periférica posible. Así la tasa de detección de la primera biopsia sería del $22 \%$, de la segunda el $10 \%$, de la tercera el $5 \%$ y de la cuarta el $4 \%$. Por lo que la estrategia actual es no realizar más de dos biopsias prostáticas. Djavan ha ideado el nomograma de Viena en el que basándose en un modelo validado matemático calcula el número de biopsias que se deben realizar en base al volumen prostático y edad en pacientes con un PSA entre 2-10 ng/ml, obteniendo una tasa de diagnóstico del 36,7\% $\%^{5}$. (Tabla 1)

El mejor conocimiento de la anatomía de la inervación prostática permite que el uso de la anestesia local sea posible. La mayoría de los nervios sensoriales aferentes proceden de los segmentos L5-L6, y en menor parte de T12-L2. El plexo prostático accede a la próstata formando las dos bandeletas neurovasculares. Los nervios

Tabla 1

Nomograma de Viena. (Djavan, et al.)

\begin{tabular}{lcccc}
\hline Volumen (cc) Edad & $\mathbf{5 0}$ & $\mathbf{5 0 - 6 0}$ & $\mathbf{6 0 - 7 0}$ & $\geq \mathbf{7 0}$ \\
\hline $20-30$ & 8 & 8 & 8 & 6 \\
$30-40$ & 12 & 10 & 8 & 6 \\
$40-50$ & 14 & 12 & 10 & 8 \\
$50-60$ & 16 & 14 & 12 & 10 \\
$60-70$ & - & 16 & 14 & 12 \\
$\geq 70$ & - & 18 & 16 & 14 \\
\hline
\end{tabular}


cavernosos llegan entre la cápsula prostática y la fascia endopélvica, localizándose entre el ápex a la base a 9-12 mm del diafragma urogenital. Los nervios capsulares emergen en dos segmentos, el primero a la altura de las vesículas seminales atravesando transversalmente la glándula en dirección caudal, el segundo llega posterolateralmente al apex 6 .

El incremento del número de biopsias implica la necesidad de utilizar una técnica anestésica y o analgésica para aumentar la tolerabilidad de la prueba.

En nuestro estudio el uso de anestésico local en las bandeletas nos ha permitido aumentar claramente el número de cilindros obtenidos, así como disminuir el dolor que conlleva la realización de esta prueba, de manera que hemos convertido una prueba casi intolerable, en una fácilmente asumible, y evitamos así la negativa del paciente a una posible rebiopsia. Coincidiendo por tanto con la mayoría de los autores que defienden el uso de anestésico local en las bandeletas, en detrimento del uso de otras posibles formas de analgesia como en nuestro caso fue la analgesia oral. Algunos autores afirman que añadir un gel de lidocaína transrectal disminuye la molestia derivada de la inyección del anestésico local en las bandeletas ${ }^{7}$.

Tobías Machado compara dos grupos de pacientes. El primero le realiza la biopsia tras la inyección en la zona de las bandeletas y ápex de $2,5 \mathrm{ml}$ de lidocaína al 1\% 10 minutos antes de la misma, en el segundo grupo se realiza la biopsia sin ningún tipo de anestesia local. La valoración del dolor se realizó mediante una escala analógica visual, considerada como uno de los mejores instrumentos de medida del dolor. En este último grupo, el $95 \%$ de los pacientes presentó algún tipo de dolor. $5 \%$ dolor leve, $20 \%$ dolor moderado, $45 \%$ dolor severo pero tolerable, y el $25 \%$ dolor intolerable. El grupo que recibió anestesia presentó dolor en el 65\% de los pacientes: $25 \%$ muy leve, $25 \%$ leve, $5 \%$ moderado y $10 \%$ severo pero tolerable, encontrándose diferencia significativa entre ambos grupos. Cabe destacar que ambos grupos refirieron dolor con la introducción del transductor, el 38\% de los pacientes del grupo anestésico y el $21 \%$ del otro grupo. Significativa es la conclusión a la que llega, ya que el $100 \%$ de los pacientes anestesiados repetiría la prueba, mientras que el 55\% del segundo grupo la rechazaria $^{8}$. El estudio de Vanni compara la inyección $5 \mathrm{ml}$ de lidocaina al $2 \%$ en las bandeletas frente a la inyección del mismo volumen de solución salina, eliminando así el efecto placebo. Curiosamente el dolor máximo en el grupo anestésico en la escala analógica fue de 6 , y de 8 en el grupo de salino, no encontrándose diferencias significativas entre ambos grupos ${ }^{9}$. Sin embargo en un trabajo publicado por Inag con el mismo planteamiento si se encontró diferencia significativa en ambos grupos a favor del de lidocaína $1 \%{ }^{10}$. Ragavan ${ }^{11}$ compara tres grupos de pacientes, al primero les bloquea las bandeletas con lidocaína $1 \%(10 \mathrm{ml})$, al segundo les administra un supositorio de $100 \mathrm{mg}$ de diclofenaco y al tercer grupo una combinación de ambos fármacos. No encontró diferencia significativa en los tres grupos a la introducción del transductor, ni en el dolor referido una hora tras la biopsia ni al día siguiente, pero sí que refleja diferencia significativa en los grupos con bloqueo de las bandeletas frente al del diclofenaco solo con respecto al dolor referido durante la biopsia. Resaltar que el $40 \%$, $14,5 \%$ y $30,6 \%$ respectivamente de los pacientes, necesitaron analgesia los 3 días posteriores a la biopsia. Un estudio multicéntrico que compara el bloqueo de las bandeletas con lidocaína o la combinación de lidocaína y bupivacaína, destaca que durante la biopsia no se encontraron niveles de analgesia diferentes en ambos grupos, pero sí trascurrida una hora tras la prueba, en la que refieren menor dolor el grupo que se benefició un anestésico de acción intermedia ${ }^{12,13}$.

Mutaguchi ${ }^{14}$ ha comparado dos técnicas anestésicas locales. Un grupo en el que anestesia las bandeletas, frente a otro en el que realiza la anestesia en múltiples inyecciones intraprostáticas, encontrando diferencia significativa entre ambos a favor del segundo grupo, pero no en la morbilidad (hematuria, hemospermia y sangrado rectal).

Finalmente otros autores abogan por el uso de analgesia intravenosa con diazepan, cyclizine y morfina ${ }^{15}$. Otros por la inhalación de $50 \%$ de óxido nitroso y oxígeno ${ }^{16}$, o la toma de analgésicos orales ${ }^{17}$ (antiinflamatorios no esteroideos, opioides). También se ha publicado el uso de enemas con lidocaína al $1 \%{ }^{18}$, defendiendo que es 
igual de efectivo el uso de estos enemas de lidocaína intrarectal, que la inyección de lidocaína al 1\% en las bandeletas en el control del dolor ${ }^{19}$. Otros estudios fomentan la utilización de anestesia tópica (EMLA) afirmando que existe diferencia significativa con respecto al grupo placebo, y que no encuentran diferencia significativa en el grupo que utiliza bloqueo de las bandeletas con lidocaína ${ }^{20,21}$. Aunque podemos ver que hay múltiples técnicas que buscan disminuir o anular el dolor, ninguno ha demostrado ser mejor que el bloqueo de las bandeletas neurovasculares con anestésico local ${ }^{22}$.

Otro punto a tener en cuenta es que la mayoría de los pacientes se queja de dolor en la introducción del transductor, que puede ser evitado con el uso de DMSO con lidocaína instilada transrectalmente 10 minutos antes de la introducción del mismo ${ }^{23}$.

\section{CONCLUSIÓN}

En nuestra experiencia la biopsia de próstata ecodirigida es una prueba muy bien tolerada bajo anestesia local en el plexo nervioso periprostático, lo cual permite realizar en el mismo acto una mayor toma de cilindros, disminuyendo el riesgo de reacción vasovagal por el dolor.

\section{REFERENCIAS}

1. Collins GN, Lloyd SN, Heihir M, McKelvie. Multiple transrectal ultrasound guided prostatic biopsies, true morbidity and patient accentance. Br J Urol 1993;71:460-463.

2. Davis M, Soler M, Kim S, Soloway MS. The procedure of transrectal ultrasound guided biopsy of the prostate: a survey of patient preparation and biopsy technique. J Urol 2002; 167:566.

3. Soloway MS Öbek C: Periprostatic local anesthesia before ultrasound guided prostate biopsy. J Urol. 2000,163:172-173.

4. Puppo P, Introini C. Ultrasound guided biopsy: the key for a correct diagnosis of prostate cancer. ESU Course"Detection of prostate cancer: the role of biopsy" San Sebastian, Spain 5 June 2005:19-37.

5. Djavan B. Prostate biopsy and the Viena Nomograms: who, how and when. An update. ESU Course"Detection of prostate cancer: the role of biopsy" San Sebastian, Spain 5 June 2005:11-18.

6. Benoit G, Mrlaud L, Meduri G, Mouzarkel NM, Quillard J, Ledroux M, et al. Anatomy of the prostatic nerves. Surg Radiol Anat 1994;16:23-29.

7. Obeck C, Ozkan B, Tunc B, Can G, Yalciin V, Solok V. Comparaison of 3 different methods of anesthesia before transrectal prostate biopsy: a prospective randomized trial. J Urol 2004;172:502-505.

8. Machado MA, Aragao AJ, Rodrigues AO, Wroclawski ER. Periprostatic local anestesia in rectal ultrasound guided prostate biopsy: is it posible to improve pain tolerante? Int Braz J Urol 2002;28:323-329.
9. Van AP, Schalal $\mathrm{CH}$, Costa RP, Sala FC. Is the periprostatic anesthetic blockade advantageous in ultrasound guided prostate biopsy ?. Int Braz J Urol 2004;30:114-118.

10. Inal G, Yazici S, Adsan O, Ozturk B, Kosan M, Cetinkaya M. Effect of periprostatic nerve blockade before transrectal ultrasound guided prostate biopsy on patient confort: a randomized placebo controlled study. Int J Urol 2004; 11(3):148-151.

11. Ragavan N, Balasubramanian PSP, DeSouza J, Marr C, Javle P. A randomized, controlled trial comparing lidocaine periprostatic nerve block, diclofenac suppository and both transrectal ultrasound guided biopsy of prostate. J Urol 2005; 174:510-513.

12. Luscombe CJ, Cooke PW. Pain during prostate biopsy. The Lancet 2004;5:363 (9424):1840-1841.

13. Rabets JC, Jones JS, Patel AR, Zippe CD. Bupivacaine provides rapid, effective periprostatic anaesthesia for transrectal prostate biopsy. BJU Int 2004;93(9):1216-1217.

14. Mutaguchi K, Shinohara K, Matsubara A, Yasumoto H, Mita $\mathrm{K}$, Usui $\mathrm{T}$. Local anestesia during 10 core biopsy of the prostate: comparison of 2 methods. J Urol 2005;173(3):742-745.

15. Fowler AW. Analgesia in prostate biopsy. The Lancet 2004; 4:364(9437):842.

16. Minankadan R, Srirangam SJ, Brown SC, O'Reilly PH, Collins GN. Nitrous oxide vs periprostatic nerve block with $1 \%$ lidocaine during transrectal ultrasound guided biopsy of the prostate: a prospective, randomised, controlled trial. J Urol 2003;170:1881-1883.

17. Bozlu M, Atici S, Ulusoy E, Canpolat B, Cayan S, Akbay E, et al. Periprostatic lidocaine infiltration and / or synthetic opioid (meperidine or tramadol) administration have no analgesic benefit during prostate biopsy. A prospective randomized double-blind placebo-controlled study comparing different methods. Urol Int 2004;72(4):308-311.

18. Trucci A, De Nuncio G, Mariani S, Pallesch G, Miano L, Tubaro A. Local anestesia reduces pain associated with transrectal prostatic biopsy. A prospective randomized study. Urol Int 2005;74(3):209-213.

19. Mallick S, Humbert M, Braud F, Fofana M, Blanchet P. Local anesthesia before transrectal ultrasound guided prostate biopsy: comparaison of 2 methods in prospective, randomized clinicl trial. J Urol 2004; 171:730-733.

20. Basar H, Basar MM, Ozcan S, Akpinar S, Batislam E. local anestesia in transrectal ultrasound guided prostate biopsy: EMLA cream as a new alternative technique. Scand J Urol Nephrol, 2005;3:130-134.

21. Galosi AB, Minardi D, Dell'atti L, Yehia M, Muzzonigro G. Tolerability of prostate transrectal biopsies using gel and local anesthetics: results of a randomized clinical trial. J Endourol 2005;19(6):738-743.

22. Nambirajan T, Woolsey S, MahendraV, Walsh IK, Lynch $\mathrm{TH}$, Keane PF. Efficacy and safety periprostatic local anesthetic injection in transrectal biopsy of the prostate: a prospective randomised study. Surgeon 2005;3(1):57.

23. Kravchiele S, Peled R, Ben-Dor D, Dorfman D, Kesari D, Cytron S. Comparison of different local anesthesia techniques during TRUS guidede biopsies: a prospective pilot study. Urology 2005;65(1):109-113.

Dra. C. Conde Redondo

E-mail: ccondere@hotmail.com

(Trabajo recibido el 7 de octubre 2006) 